\title{
Oral administration of ampelopsin protects against acute brain injury in rats following focal cerebral ischemia
}

\author{
XIAO-LI YE ${ }^{1}$, LING-QUN LU², WEI LI ${ }^{2}$, QI LOU ${ }^{2}$, HONG-GANG GUO ${ }^{2}$ and QIAO-JUAN SHI ${ }^{2}$ \\ ${ }^{1}$ Department of Pharmacy, The Children's Hospital of Zhejiang University School of Medicine, Hangzhou, \\ Zhejiang 310003; ${ }^{2}$ Experimental Animal Center, Zhejiang Academy of Medical Sciences, \\ Hangzhou, Zhejiang 310013, P.R. China
}

Received August 11, 2015; Accepted December 19, 2016

DOI: $10.3892 /$ etm.2017.4197

\begin{abstract}
Ampelopsin (AMP) is isolated from the Chinese medicinal herb Ampelopsis grossedentata (Hand-Mazz) and has been associated with numerous biological and pharmacological activities. However, it is not clear whether AMP has a direct protective effect on cerebral ischemia reperfusion injury. Therefore, the present study investigated its role in acute brain injury following focal cerebral ischemia in rats. The current study induced transient focal cerebral ischemia by performing middle cerebral artery occlusion (MCAO) for $60 \mathrm{~min}$, followed by $24 \mathrm{~h}$ of reperfusion. Rats were exposed to 40, 80 and $160 \mathrm{mg} / \mathrm{kg}$ AMP by oral administration $30 \mathrm{~min}$ prior to MCAO and the cysteinyl leukotriene receptor 1-antagonist, pranlukast $(0.1 \mathrm{mg} / \mathrm{kg}$, i.p.) was used as a positive control. Neurological deficit scores were observed and an inclined board test was used to assess behavioral dysfunction. The coronal slices were stained with 3,5-triphenyltetrazolium chloride to determine the infarct volume and brain edema. Neuronal morphology was assessed in brain sections stained with cresyl violet and degenerating neurons were identified using Fluoro-Jade B staining. Blood-brain barrier permeability was determined with immunoglobulin (Ig)G immunohistochemistry. Interleukin (IL)-1 $\beta$, tumor necrosis factor- $\alpha$ (TNF- $\alpha$ ) in serum and cerebrospinal fluid were measured using ELISA kits. AMP at 80 and $160 \mathrm{mg} / \mathrm{kg}$ attenuated neurological deficits, reduced infarct volume, brain edema, $\operatorname{IgG}$ exudation and neuron degeneration and loss. Similar to pranlukast, AMP also inhibited the MCAO-induced IL-1 $\beta$ and TNF- $\alpha$ release. Thus, AMP has a neuroprotective effect on acute brain injury following focal cerebral ischemia in rats at an effective oral dose of $80-160 \mathrm{mg} / \mathrm{kg}$. The results
\end{abstract}

Correspondence to: Dr Qiao-Juan Shi, Experimental Animal Center, Zhejiang Academy of Medical Sciences, 182 Tianmushan Road, Hangzhou, Zhejiang 310013, P.R. China

E-mail: shiqiaojuan@163.com

Key words: ampelopsin, middle cerebral artery occlusion, neuroprotection, pranlukast of the current study indicate a therapeutic role for AMP in the treatment of ischemic stroke.

\section{Introduction}

Stroke is a major life-threatening disease with a worldwide incidence of approximately two in 1,000 per year, and a annual $8 \%$ mortality rate (1). Despite the development of shock therapy and the application of advanced thrombolytic agents and intravascular procedures, clinical therapy of the debilitating disorder remains unsatisfactory $(2,3)$. Therefore, neuroprophylaxis against stroke has received more attention.

Ampelopsin (2R,3R)-3,5,7-trihydroxy-2-(3,4,5trihydroxyphenyl)-2,3-dihydrochromen-4-one; AMP; Fig. 1), also referred to as dihydromyricetin, is the primary bioactive component and one of the most common flavonoids isolated from the tender stem and leaves of the Chinese medicinal herb Ampelopsis grossedentata (Hand-Mazz) W.T. Wang, which is typically used to make an infusion called Rattan tea $(4,5)$. AMP is collected from the tender stem and leaves of the species, which contain $>27 \%$, and the cataphylls, which contains $>40 \%(6,7)$.

AMP exhibits a number of biological and pharmacological properties including antimicrobial, anti-inflammatory, antioxidative, anti-hypertensive, hepatoprotective and anticarcinogenic activities in addition to providing cough relief (5,8-13). Previous studies have demonstrated that AMP not only exhibits acetylcholinesterase inhibitory activities, suggesting anti-Alzheimer effects (14), but also decreases the aggregation of $\beta$-amyloid peptide, which is a key compound in the induction of Alzheimer's disease (15). These results suggest a role of AMP in protecting against brain cell dysfunction. AMP protects PC12 cells from $\mathrm{H}_{2} \mathrm{O}_{2}$-induced apoptosis by activating the extracellular-signal related kinase (ERK) and protein kinase $\mathrm{B}(\mathrm{Akt})$ signaling pathways and upregulation of heme oxygenase-1 (12). Additionally, the protective effects of the leaf and stem of Vitis amurensis against neuronal injury induced by middle cerebral artery occlusion (MCAO) followed by reperfusion in rats and against glutamate-induced excitotoxicity in cultured rat cortical neurons have been reported $(10,12)$. AMP is reportedly one of the active components contributing to the neuroprotective effect of $V$. amurensis against glutamate-induced neurotoxicity (10). However, based 
on the previous studies described, it is not clear whether AMP has a direct or indirect protective effect against cerebral ischemia reperfusion injury.

Upregulation of cerebral inflammatory cytokines, disruption of the blood-brain barrier (BBB), activation of systemic lymphocytes, local microglia and astrocytes substantially contribute to ischemic damage (16-19). Rattan tea extraction has been indicated to reduce carrageenan-induced acute inflammation in vivo (20) and AMP inhibited the production of nitric oxide in lipopolysaccharide (LPS)-challenged RAW264.7 macrophages (21). Previous studies have confirmed that AMP reduced LPS/toll-like receptor 4-mediated inflammation characterized by nitric oxide biosynthesis and pro-inflammatory cytokines [interleukin (IL)-1 $\beta$, tumor necrosis factor- $\alpha$ (TNF- $\alpha$ ) and IL-6] production in RAW264.7 macrophages, mediated by the reactive oxygen species (ROS), Akt, inhibitor of nuclear factor-kB (IKK) and nuclear factor $\kappa B(N F-\kappa B)$ signaling pathways (9). However, whether AMP inhibits inflammatory responses such as the generation of inflammatory cytokines and destruction of the BBB remains unknown.

The aims of the current study were to systematically evaluate whether AMP protected against acute brain injury following focal cerebral ischemia in rats and to elucidate the mechanisms underlying AMP activity. The results of the present study provide experimental evidence to support the development of AMP as an effective and safe candidate for the prevention and/or therapy of cerebral ischemia. The current study used the cysteinyl leukotriene receptor 1 $\left(\right.$ CysLT $\left._{1} \mathrm{R}\right)$-selective antagonist, pranlukast, an anti-inflammatory agent as a positive control, as it has been reported to protect against cerebral ischemia $(17,22-24)$.

\section{Materials and methods}

Animals. A total of 92 male Sprague-Dawley rats weighing 250-300 $\mathrm{g}$ and 10-12 weeks old were supplied by the Experimental Animal Center, Zhejiang Academy of Medicine Sciences (Hangzhou, China; Certificate no. SCXK (Zhe) 2014-0001). The animals were housed in a controlled temperature of $20-24^{\circ} \mathrm{C}$, under a 12 -h light/dark cycle with ad libitum access to food and water with the exception of preoperative fasting. Procedures involving animals and their care were performed in accordance with the National Institutes of Health Guide for the Care and Use of Laboratory Animals. All experimental protocols were approved by the Ethics Committee of Laboratory Animal Care and Welfare at the School of Medicine, Zhejiang University (Hangzhou, China). Every effort was made to minimize the number of animals used and their suffering.

Chemicals. AMP with $95 \%$ purity was donated by Dr Jiye-Zhang (College of Pharmacy. Xi'an Jiaotong University, Xi'an, China), 3,5-triphenyltetrazolium chloride (TTC) was purchased from Sigma-Aldrich (Merck KGaA, Darmstadt, Germany). Fluoro-Jade B was purchased from Merck Millipore (Merck KGaA). Pranlukast was a kind gift from Dr. Masami Tsuboshima (Ono Pharmaceutical Co. Ltd, Osaka, Japan). Paraformaldehyde, sucrose, cresyl violet and chloral hydrate were purchased from Sigma-Aldrich; Merck
KGaG (Darmstadt, Germany). Biotinylated anti-rat IgG antibody (catalogue no. 20141021) and streptavidin horse radish peroxidase (catalogue no. 20140815) were from Vector laboratories, Inc., (Burlingame, CA, USA), repacked and sold by Zhongshan Goldenbridge Biotechnology Co., Ltd (Beijing, China).

Transient focal cerebral ischemia induction. Transient focal cerebral ischemia was induced using a modified method of MCAO (25) according to a previously reported method (26). The 92 rats were anaesthetized with an intraperitoneal injection of chloral hydrate $(400 \mathrm{mg} / \mathrm{kg})$ and placed in dorsal recumbency. The left common carotid artery, external carotid artery (ECA) and internal carotid artery (ICA) were isolated. A 4-0 monofilament nylon suture (Beijing Sunbio Biotech Co., Ltd., Beijing, China) with a round poly-L-lysine coated tip was inserted from the ECA into the ICA $18-19 \mathrm{~mm}$ until a slight resistance was felt, which indicated that the suture had blocked the origin of the left middle cerebral artery (MCA). The MCA was occluded for $60 \mathrm{~min}$ and the suture was withdrawn to allow reperfusion. The ECA was ligated and the incision was closed. Sham-operated rats $(n=12)$ were manipulated similarly; the monofilament was inserted to a depth of $1.0-2.0 \mathrm{~mm}$ without occluding the MCA. Body temperature was maintained at $36-37^{\circ} \mathrm{C}$ with a warming platform (Kent Scientific Services, West Malling, UK). Following surgery, rats were maintained for $\sim 2 \mathrm{~h}$ in a warm box heated by lamps to maintain body temperature.

The rats were randomized into six groups: Sham $(\mathrm{n}=12)$, saline ( $\mathrm{n}=17)$, AMP [40 ( $\mathrm{n}=16), 80(\mathrm{n}=15)$ or $160(\mathrm{n}=15) \mathrm{mg} / \mathrm{kg}$, $1 \mathrm{ml}$ per $100 \mathrm{~g}$ bodyweight per os (p.o.) (12)] and pranlukast $(\mathrm{n}=17,0.1 \mathrm{mg} / \mathrm{kg}$ intraperitoneally (i.p.)). Due to a $20-30 \%$ mortality during the surgical procedure, the final number of each surgery group was $n=12$. AMP and pranlukast were administered to the rats $(\mathrm{n}=12) 30 \mathrm{~min}$ prior to and $30 \mathrm{~min}$ following MCAO. Pranlukast was used as a positive control. The injuries were evaluated $24 \mathrm{~h}$ following reperfusion. An equal volume $(10 \mathrm{ml} / \mathrm{kg})$ of saline was administered orally as the control.

Cerebrospinal fluid (CSF) collection. CSF was collected as previously described $(27,28)$ and modified as follows: Animals were anesthetized with chloral hydrate $(400 \mathrm{mg} / \mathrm{kg})$ via i.p. injection. The fur on the neck region of the rat was removed using an oster clipper. The anesthetized rat was placed in a stereotaxic frame (Stoelting Inc., Wood Dale, IL, USA) and secured with ear bars. The position of the animal's head was maintained downward at $\sim 45^{\circ}$ using straps and clamps. Using tweezers and scissors, the atlanto-occipital membrane between the occipital protuberance and the spine of the atlas was exposed. A needle connected to a draw syringe was inserted horizontally and centrally into the cisterna magna for CSF collection without making any incision at this region. Once a change in resistance was felt along the direction of insertion, the colorless CSF was gently aspirated through the needle into the syringe. The color of the CSF was closely observed to avoid any blood contamination. In the process of sampling the CSF, the pumping strength was controlled to avoid blood contamination. CSF was ejected into $0.5 \mathrm{ml}$ Eppendorf tubes and frozen at $-80^{\circ} \mathrm{C}$. The volume of CSF ranged from 60-150 $\mu \mathrm{l}$ in all the samples. 
Behavioral assessments. Neurological deficit scores were evaluated $24 \mathrm{~h}$ following MCAO, as previously described (22): 0 , no deficit; 1 , failure to extend left forepaw fully; 2 , circling to the left; 3 , falling to the left; and 4, no spontaneous walking with a depressed level of consciousness. An inclined board test was used to assess balance and coordination based on a modified method (29). Rats were placed on a board $(50 \times 30 \mathrm{~cm})$ and stabilized. The board was then inclined from the horizontal to a vertical plane. The degree at which the animal fell from the board (holding angle) was recorded. The assessment was repeated three times and the average degree was used. An examiner blinded to the experiment groups completed all behavioral assessments.

Infarct volume and brain edema. Following behavioral assessments, 36 rats were sacrificed by decapitation, following anesthetic with chloral hydrate $(400 \mathrm{mg} / \mathrm{kg})$ via i.p. injection; the brains were removed rapidly and stored at $-20^{\circ} \mathrm{C}$ for $10 \mathrm{~min}$. The brain was sliced into $2-\mathrm{mm}$ coronal sections and stained with $0.5 \% \mathrm{TTC}$ solution at $37^{\circ} \mathrm{C}$ for $20 \mathrm{~min}$ in the dark, then fixed at $25^{\circ} \mathrm{C}$ in $4 \%$ buffered formalin overnight. Images of the stained slices were obtained using a FinePix S602 Zoom digital camera (Fujifilm, Tokyo, Japan). The total infarction volume was measured as the sum of the infarcts of each of the six sections. Infarct areas of all sections were added to obtain the total infarct area, which was multiplied by the thickness of the brain sections. Edema was evaluated indirectly as the percentage increase of ischemic vs. non-ischemic hemisphere volume. To partially compensate for the effects of edema, the corrected infarct area was determined as previously described (30) Corrected infarct area $=$ Measured infarct area $\mathrm{x}$ (1 - [(ipsilateral hemisphere area - contralateral hemisphere area) / contralateral hemisphere area]).

Histopathology and immunohistochemistry. From a second group, 36 rats were anesthetized with chloral hydrate (400 mg/kg) via i.p. injection $24 \mathrm{~h}$ following MCAO and perfused transcardially with $4 \%$ paraformaldehyde following a pre-wash with saline prior to being sacrificed by decapitation. The brain was then removed, fixed at $25^{\circ} \mathrm{C}$ in $4 \%$ paraformaldehyde overnight and transferred to $30 \%$ sucrose and incubated for 5 days at $25^{\circ} \mathrm{C}$. The whole brain was imaged using a FinePix S602 Zoom digital camera to record the changes on the surface. Subsequently, frozen coronal sections were sliced into $10-\mu \mathrm{m}$ sections using a CM 1900 cryomicrotome (Leica Microsystems GmbH, Wetzlar, Germany). The sections were stained with cresyl violet and Fluoro-Jade B prior to use in immunohistochemical examination.

Cresyl violet staining. Cresyl violet is a basic aniline dye used to stain RNA blue in order to highlight important structural features of neurons, specifically in brain and spinal cord tissue. The brain sections were deparaffinized by soaking in a 1:1 $100 \%$ alcohol $/ 100 \%$ chloroform mixture for $15 \mathrm{~min}$ followed by $15 \mathrm{~min}$ in $100 \%$ alcohol and $95 \%$ alcohol/5\% deionized $\mathrm{H}_{2} \mathrm{O}$ mixture for rehydration. The sections were transferred to $0.1 \%$ cresyl violet solution for $10 \mathrm{~min}$, rinsed quickly in distilled water and differentiated in $95 \%$ ethyl alcohol for $30 \mathrm{~min}$ to allow for the best contrast. The sections were dehydrated in $100 \%$ alcohol, immersed in xylene and cover-slipped. The stained sections were observed under a BX-51 fluorescence microscope (Olympus Corporation, Tokyo, Japan). The neurons in the cortex, both the ischemic core and the boundary zone, were counted using ImageJ software (National Institutes of Health, Bethesda, MA, USA).

Fluoro-Jade B staining. Fluoro-Jade B has high affinity for degenerating apoptotic and necrotic neurons, exhibiting a green fluorescence. Fluoro-Jade B staining was performed according to a modified protocol (31). Briefly, the brain sections were air-dried on a slide warmer at $50^{\circ} \mathrm{C}$ and immersed in $1 \%$ sodium hydroxide in $80 \%$ alcohol for $5 \mathrm{~min}$ followed by $2 \mathrm{~min}$ in $70 \%$ alcohol and $2 \mathrm{~min}$ in distilled water. The sections were then transferred to $0.06 \%$ potassium permanganate for $15 \mathrm{~min}$, followed by $2 \mathrm{~min}$ in distilled water. The sections were transferred to $0.0004 \%$ Fluoro-Jade staining solution for $30 \mathrm{~min}$ and rinsed with distilled water for $2 \mathrm{~min}$. Following drying, the sections were immersed in xylene, cover-slipped with mounting medium (Dako, Glostrup, Denmark) and examined using a BX-51 fluorescence microscope. ImageJ was used to count the degenerating neurons.

Endogenous immunoglobulin (Ig)G immunostaining. Endogenous IgG immunostaining was performed to detect the permeability of the BBB (32). Sections (1.8-2.0 mm caudal from bregma) were incubated with biotinylated anti-rat IgG antibody (\#20141021; 1:200) for $2 \mathrm{~h}$ at $37^{\circ} \mathrm{C}$, and with streptavidin horse radish peroxidase (\#20140815; 1:200; Vector laboratories, Inc., Burlingame, CA, USA) for $2 \mathrm{~h}$ at $37^{\circ} \mathrm{C}$. Finally, the sections were exposed for $2 \mathrm{~min}$ to $0.05 \%$ 3,3'-diaminobenzidine and $0.03 \% \mathrm{H}_{2} \mathrm{O}_{2}$. The sections were then digitized, the optical gray scales in the immunostained sections were detected with ImageJ $1.37 \mathrm{c}$ (National Institutes of Health, Bethesda, MD, USA). IgG exudation was evaluated by determining the stained area and as percentage increase of the gray scales of the ischemic hemisphere: (Gi-G0)/G0x100\%, where Gi is the grayscale intensity of the ischemic hemisphere and G0 is the grayscale intensity of the contralateral uninjured hemisphere. The total IgG extravasation area of all coronal sections was evaluated using the same image analysis program.

Cytokine assay by ELISA. The serum and CSF were collected $24 \mathrm{~h}$ following $\mathrm{MCAO}$ and were frozen at $-80^{\circ} \mathrm{C}$ until analysis. The cytokines IL- $1 \beta$ and TNF- $\alpha$ in the medium were measured using commercial ELISA kits for IL-1 $\beta$ (Rat IL-1 $\beta$ ELISA kit, \#140609) and TNF- $\alpha$ (Rat TNF-alpha ELISA kit, \#140528; Changzhou Lianke Chemical Technology Co., Ltd., Hangzhou, China) according to the manufacturers' protocol.

Statistical analysis. Values are expressed as the mean \pm standard error of the mean. Statistical analyses were performed using one-way analysis of variance, followed by Newman-Keuls post-hoc multiple comparison and nonparametric Kruskal-Wallis test for neurological deficit scores using Prism software, version 4.02 (GraphPad Software Inc., La Jolla, CA, USA). P $<0.05$ was considered to represent a statistically significant difference. 


\section{Results}

AMP improves neurological score and holding angle in MCAO-injured rats. No mortality was observed in sham-operated rats. The mortality of ischemic rats was $22 \%$ in each group $(n=12) 24 \mathrm{~h}$ following ischemia. No significant difference in mortality rates was identified between the groups.

Sham-operated rats demonstrated no neurological deficit throughout the observation period. Saline-treated ischemic rats exhibited significant neurological deficits $24 \mathrm{~h}$ following MCAO $(\mathrm{P}<0.01)$ compared with the saline control. In rats treated with 80 and $160 \mathrm{mg} / \mathrm{kg}$ AMP and the pranlukast $(0.1 \mathrm{mg} / \mathrm{kg})$ groups of rats, neurological deficits were significantly reduced $(\mathrm{P}<0.01$; Fig. $2 \mathrm{~A})$.

In the inclined board test, the inclination significantly decreased $24 \mathrm{~h}$ following MCAO in saline-treated ischemic rats compared with sham control $(\mathrm{P}<0.01)$. Exposure to AMP (80 and $160 \mathrm{mg} / \mathrm{kg}$ ) and pranlukast significantly increased the holding angles in the inclined board test $(\mathrm{P}<0.01$ and $\mathrm{P}<0.05$, respectively; Fig. 2B).

AMP reduces $M A O C$-induced infarct volume and mitigates the increase in ischemic hemisphere volume. Ischemia induced brain lesion and edema $24 \mathrm{~h}$ following MCAO. TTC-stained coronal slices exhibited clear lesion or infarct areas in the ischemic hemispheres (Fig. 3A). AMP (80 and $160 \mathrm{mg} / \mathrm{kg}$ ) reduced the lesion volume by 30.7 and $44.6 \%$, respectively, compared with saline group, $24 \mathrm{~h}$ following MCAO (Fig. 3B). There was a reduction in the ratio of the left/right hemispheric ratio (edema) at $24 \mathrm{~h}$ following $\mathrm{MCAO}$ of 43.2 and $54.1 \%$, following treatment with 80 and $160 \mathrm{mg} / \mathrm{kg}$ AMP, respectively. Pranlukast exerted similar effects (Fig. 3C).

AMP inhibited MCAO-induced neuronal loss in the ischemic core. Cresyl violet staining of coronal slices indicated neuronal damage in the temporoparietal cortex of ischemic hemispheres (Fig. 4A), as a reduction in nissl bodies, shrunken and deep-stained cell bodies (Fig. 4B). Neuronal dendrites were significantly decreased $24 \mathrm{~h}$ following reperfusion $(\mathrm{P}<0.05)$. AMP (80 and $160 \mathrm{mg} / \mathrm{kg})$ in addition to pranlukast $(1 \mathrm{mg} / \mathrm{kg}$, i.p.) ameliorated neuronal loss in the ischemic core (Fig. 4C) and the boundary zone (Fig. 4D) adjacent to the infarcted area.

AMP mitigates MCAO-induced neuronal degeneration in the ischemic core and boundary zone of the rat brain. The density of Fluoro-Jade B-positive degenerating neurons in the cortex III and IV layers of the infarcted hemisphere increased gradually $24 \mathrm{~h}$ following MCAO, specifically in the ischemic core. A small number of degenerating neurons were observed in the sham-operated rats (Fig. 5A). In addition to AMP (80 and $160 \mathrm{mg} / \mathrm{kg}$ ), pranlukast decreased the number of degenerating neurons in the ischemic core (Fig. 5B) and boundary zone (Fig. 5C) $24 \mathrm{~h}$ following MCAO. However, the most marked reduction in the number of degenerating neurons was induced by $80 \mathrm{mg} / \mathrm{kg}$ AMP, while $160 \mathrm{mg} / \mathrm{mg}$ AMP and $0.1 \mathrm{mg} / \mathrm{kg}$ pranlukast treatments had a similar and less marked effect.

AMP decreases MCAO-induced elevation of Ig $G$ exudation. The BBB was disrupted $24 \mathrm{~h}$ following reperfusion, as $\mathrm{IgG}$<smiles>O=C1c2c(O)cc(O)cc2OC(c2cc(O)c(O)c(O)c2)C1O</smiles>

Figure 1. Chemical structure of ampelopsin.

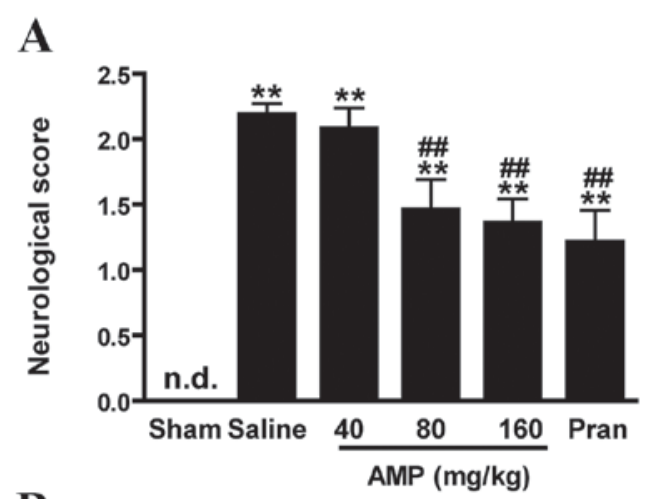

B

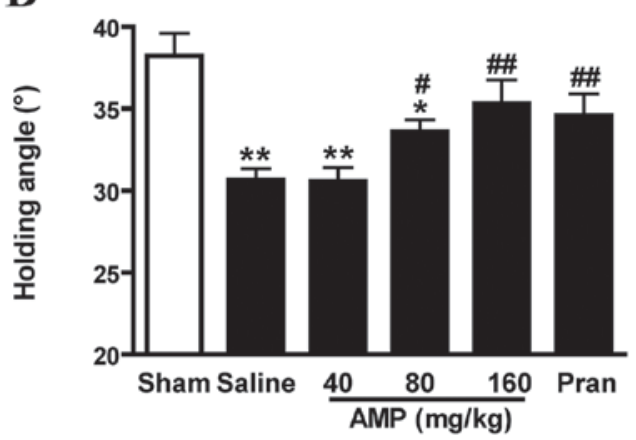

Figure 2. Effects of AMP and pran on neurological dysfunction $24 \mathrm{~h}$ following middle cerebral artery occlusion in rats. (A) Neurological deficit scores increased and (B) the holding angle in the inclined board test decreased $24 \mathrm{~h}$ following reperfusion. AMP (80 and $160 \mathrm{mg} / \mathrm{kg}$, per os (p.o.)) and pran $(0.1 \mathrm{mg} / \mathrm{kg}$, intraperitoneally (i.p.)) significantly improved the neurological dysfunction $(\mathrm{n}=12)$. ${ }^{*} \mathrm{P}<0.05$ and ${ }^{* *} \mathrm{P}<0.01$ vs. sham, ${ }^{\#} \mathrm{P}<0.05$ and ${ }^{\# \#} \mathrm{P}<0.01$ vs. ischemic control, analyzed using one-way analysis of variance for holding angle and nonparametric Kruskal-Wallis test for neurological deficit scores. AMP, ampelopsin; Pran, pranlukast; n.d., not-detectable.

exudation was detected in the ischemic hemispheres however, in the sham operated rats the level of $\mathrm{IgG}$ exudation was almost undetectable (Fig. 6A). IgG exudation was significantly increased $24 \mathrm{~h}$ following reperfusion in the $0 \mathrm{mg} / \mathrm{kg}$ AMP control group compared with the sham group $(\mathrm{P}<0.01$; Fig. 6B). AMP (40, 80 and $160 \mathrm{mg} / \mathrm{kg}$ ) and pranlukast significantly inhibited the increase of $\mathrm{IgG}$ exudation at $24 \mathrm{~h}$ following reperfusion by $33.4,48.1,55.6$ and $46.7 \%$, respectively $(\mathrm{P}<0.01$; Fig. 6B), compared with the $0 \mathrm{mg} / \mathrm{kg}$ AMP control group, indicating an inhibitory effect on BBB disruption.

$A M P$ reduces the $M C A O$-induced expression of $I L-1 \beta$ and $T N F-\alpha$ in rat serum and CSF. Furthermore, the increased serum level of MCAO-induced IL-1 $\beta$ and TNF- $\alpha 24 \mathrm{~h}$ following reperfusion was significantly decreased following 
A
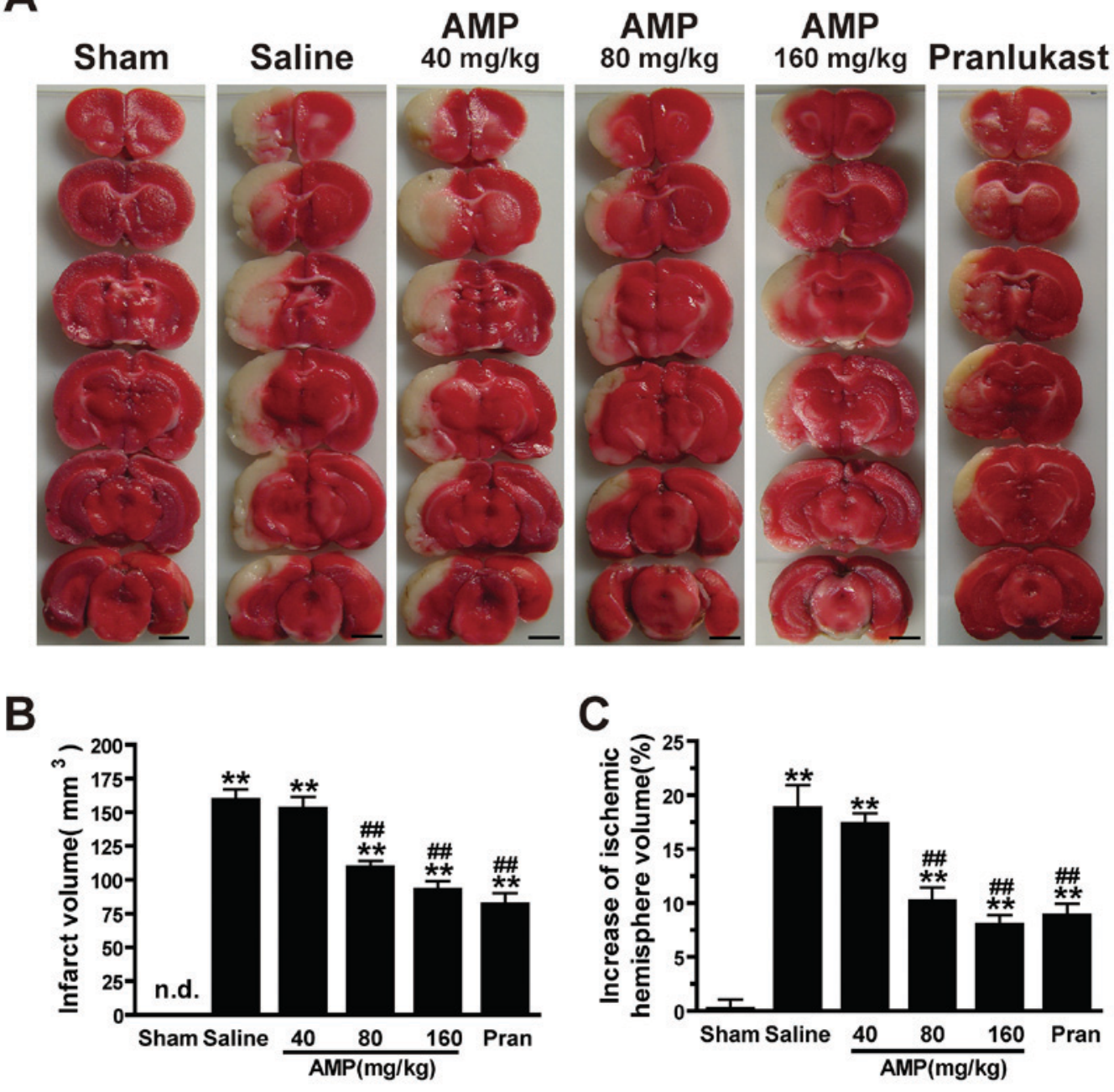

Figure 3. Effects of ampelopsin and pran on infarct volume and brain edema $24 \mathrm{~h}$ following MCAO in rats. (A) Images of 3,5-triphenyltetrazolium chloride-stained coronal slices indicate brain lesions $24 \mathrm{~h}$ following MCAO. (B) Infarct volume and (C) percent increase in ischemic hemisphere volume were reduced dose-dependently by AMP (80 and $160 \mathrm{mg} / \mathrm{kg}$, p.o.) and pran $\left(0.1 \mathrm{mg} / \mathrm{kg}\right.$, i.p.) treatment $(\mathrm{n}=6)$. ${ }^{*} \mathrm{P}<0.05$ and ${ }^{* * *} \mathrm{P}<0.01 \mathrm{vs}$. sham, ${ }^{\#} \mathrm{P}<0.05$ and ${ }^{\# \#} \mathrm{P}<0.01$ vs. ischemic control (vehicle), analyzed by one-way analysis of variance. Scale bar, 4 mm. AMP, ampelopsin; Pran, pranlukast; MCAO, middle cerebral artery occlusion; n.d., not detectable.

treatment with AMP $(160 \mathrm{mg} / \mathrm{kg})$. Pranlukast $(0.1 \mathrm{mg} / \mathrm{kg})$ similarly reduced the concentration of IL-1 $\beta$ and TNF- $\alpha$ in the rat serum and CSF (Fig. 7).

\section{Discussion}

The present study, to the best of our knowledge, is the first to indicate the neuroprotective effect of AMP on acute brain injury following focal cerebral ischemia in rats. AMP attenuated the neurological deficits and neuronal loss in addition to reducing the lesion volume, edema, neuronal degeneration, $\operatorname{IgG}$ exudation and the release of TNF and IL- $1 \beta$ following MCAO. This protective effect was similar to that of the $\mathrm{CysLT}_{1} \mathrm{R}$ antagonist pranlukast, an anti-inflammatory agent (17,22-24). These findings support the hypothesis that AMP reduced ischemic injury in the brain, at least in the acute phase.

The severity of neurological deficits is associated with the size of brain infarct and edema. The recovery of neurological function is the primary determinant in the treatment of cerebral ischemia/reperfusion injury (33). Following $60 \mathrm{~min}$ of MCAO and $24 \mathrm{~h}$ reperfusion, rats exhibited serious neurological dysfunction including increased neurological deficit scores and decreased holding angles in the inclined board test (34). AMP administration improved the behavioral assessment in a dose-dependent fashion, suggesting a neuroprotective effect.

Neuronal cells are particularly vulnerable to cerebral ischemia. Therefore, in acute ischemia, neuronal injury is predominantly attributed to factors including inflammation, oxidative stress, energy failure and excitotoxicity $(18,35)$. Neuronal degeneration and necrosis have been identified to be associated with behavioral deficits $(36,37)$. The present study demonstrated that not only neuronal loss and degeneration but also the behavior assessment was improved following treatment with AMP. The neuroprotective effect of AMP was apparent in the ischemic core and the boundary zone. The results of the current study were consistent with AMP protection of neuron-like $\mathrm{PC} 12$ cells against $\mathrm{H}_{2} \mathrm{O}_{2}$-induced cytotoxicity via ERK and Akt signaling pathways (12).

BBB breakdown is a common feature in cerebral ischemia and is known to occur due to vascular damage, within $3-5 \mathrm{~h}$ and persists for a number of days (38). Therefore, rapid protection against BBB dysfunction is critical in any therapeutic 
A

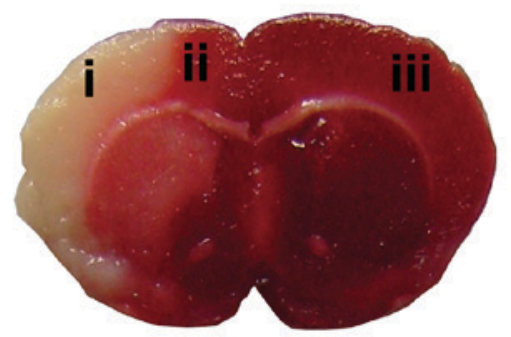

\section{i: Ischemic core}

\section{ii: Boundary zone}

iii: Contralateral

B
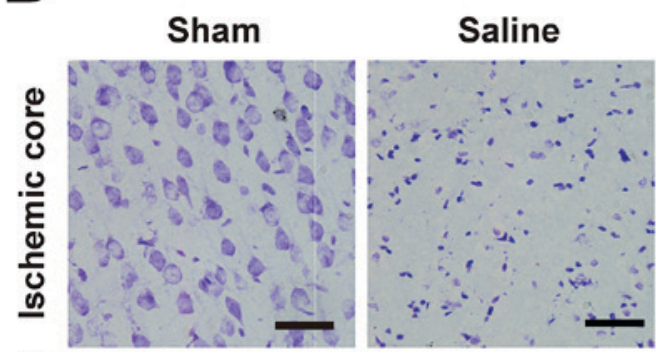

$\operatorname{AMP}(160 \mathrm{mg} / \mathrm{kg})$
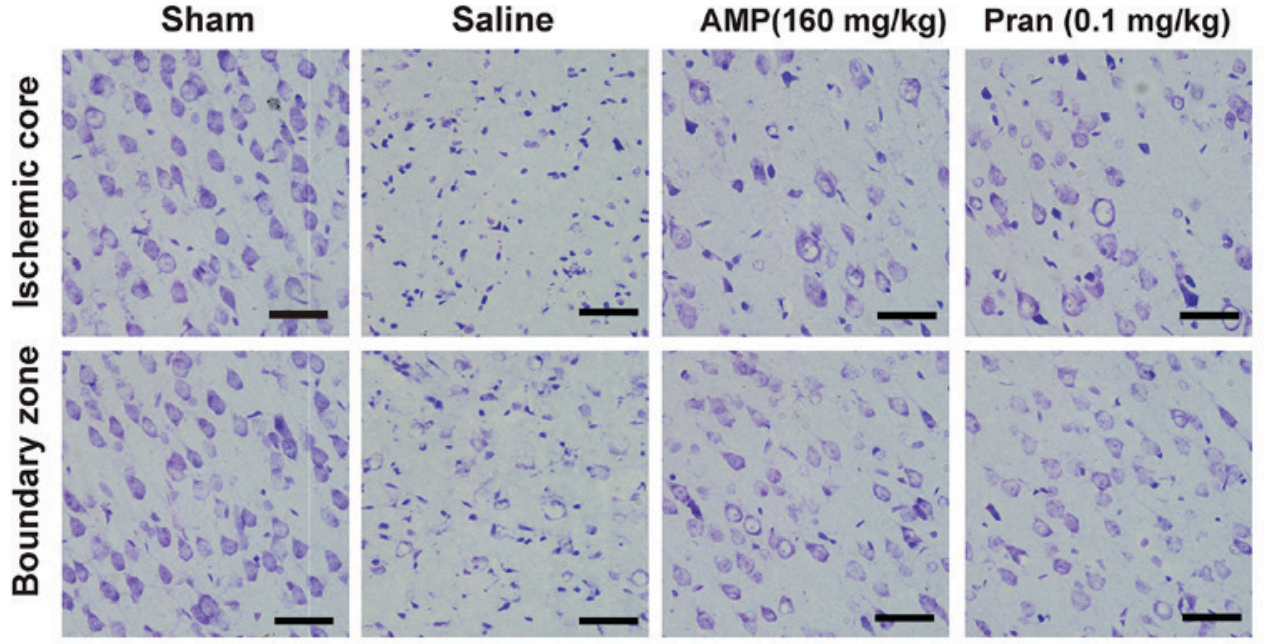

C

Ischemic core

D
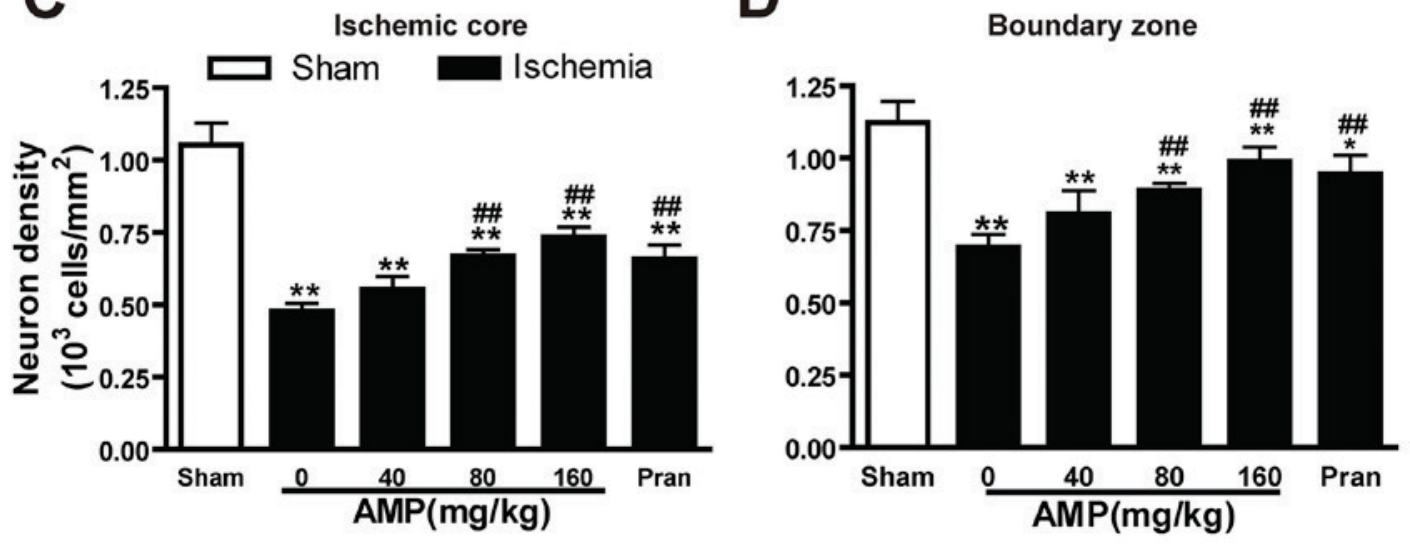

Figure 4. Effects of AMP and pran on histopathological changes and neuron density in the cortex $24 \mathrm{~h}$ following middle cerebral artery occlusion in rats. (A) Sampling regions for micro-imaging examination. (B) Cresyl violet staining indicated altered neuronal density in the ischemic core and boundary zone $24 \mathrm{~h}$ following reperfusion. Cresyl violet-stained neurons in the (C) ischemic core and (D) boundary zone. Neuronal loss in the ischemic core and boundary zone was ameliorated by AMP $\left(80\right.$ and $160 \mathrm{mg} / \mathrm{kg}$, p.o.) and pran $\left(0.1 \mathrm{mg} / \mathrm{kg}\right.$, i.p.) treatment $(\mathrm{n}=6)$. ${ }^{*} \mathrm{P}<0.05$ vs . sham ${ }^{* * *} \mathrm{P}<0.01$ vs. sham, ${ }^{\#} \mathrm{P}<0.05$ and ${ }^{\# \#} \mathrm{P}<0.01$ vs. ischemic control, analyzed by one-way analysis of variance. Scale bar, $50 \mu \mathrm{m}$. AMP, ampelopsin; Pran, pranlukast.

intervention to minimize neuronal injury. The data of the current study demonstrates that AMP reduces cerebral IgG extravasation following focal cerebral ischemia. This suggests that AMP improves cerebral ischemia injury by attenuating BBB breakdown, by decreasing the cerebral edema and neuronal loss and degeneration.

Inflammation is a significant contributing factor to the pathology of brain damage following ischemic insult mediated by the activation of microglia and astrocytes, BBB disruption, production of various cytokines such as TNF- $\alpha$ and IL-1 $\beta(16,18)$. Systemic administration of anti-inflammatory agents reduces brain edema and infarct sizes, improves neurological deficit and regulates cytokine expression in the cortex (17,22-24). In a prior study, AMP decreased the expression of LPS-induced pro-inflammatory cytokines TNF- $\alpha$, IL-1 $\beta$, IL-6 and pro-inflammation mediator nitric oxide via the direct downregulation of intracellular ROS levels and Akt phosphorylation indirectly, which subsequently results in suppression of NF- $\kappa \mathrm{B}$ activation in RAW264.7 macrophages (9). Furthermore, Rattan tea extract has been suggested to reduce carrageenan-induced acute inflammation in vivo (20). AMP prevented oxidative stress in vivo due to ROS generated 
A
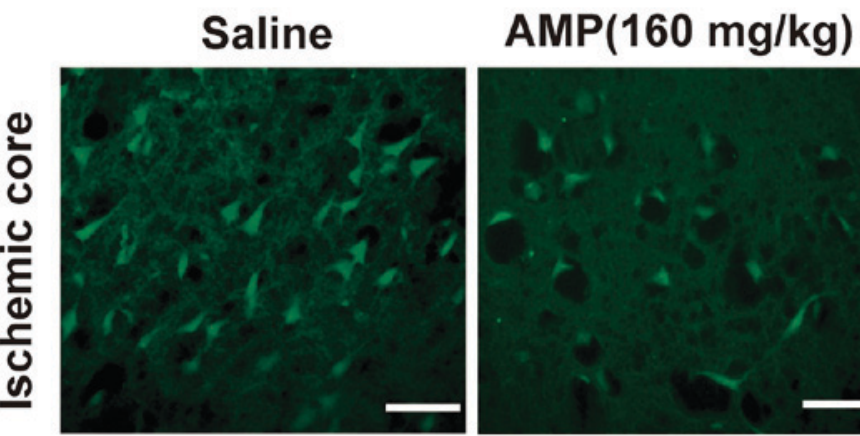

$\operatorname{Pran}(0.1 \mathrm{mg} / \mathrm{kg})$
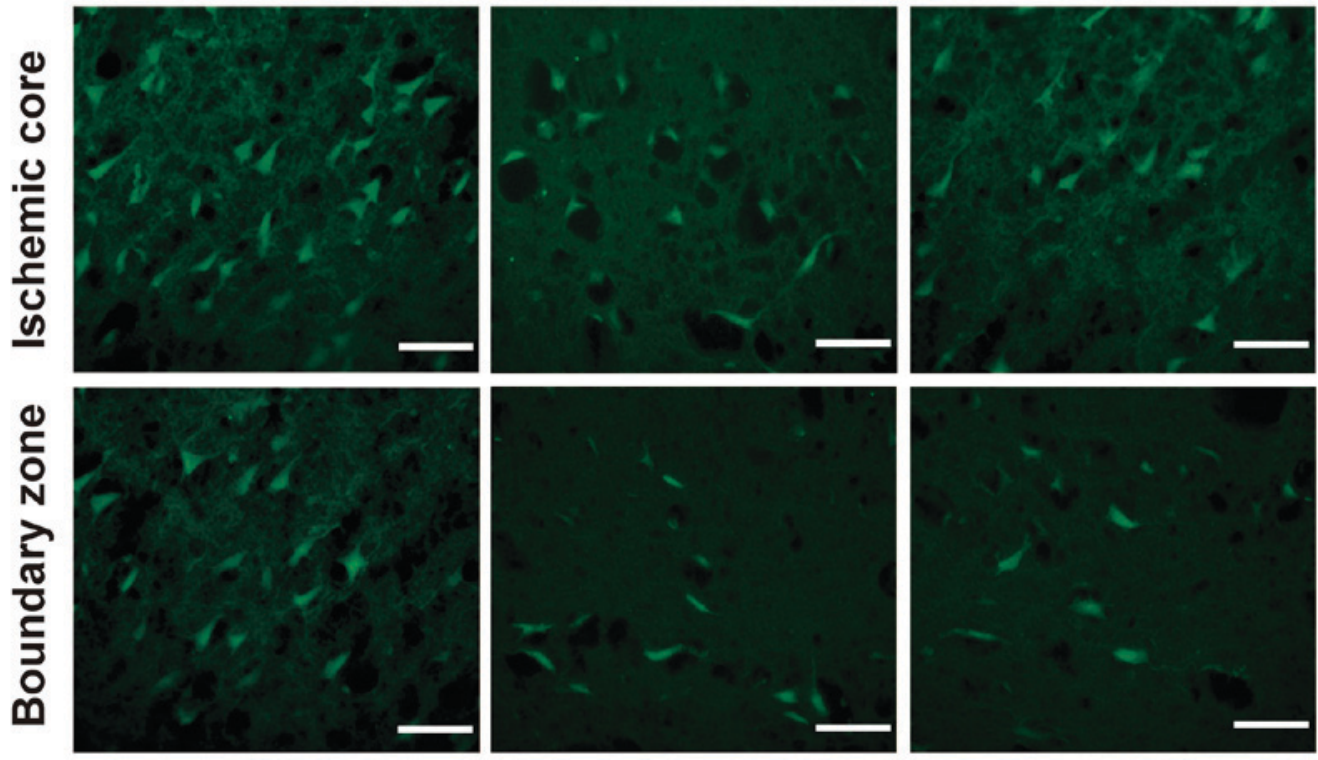

B
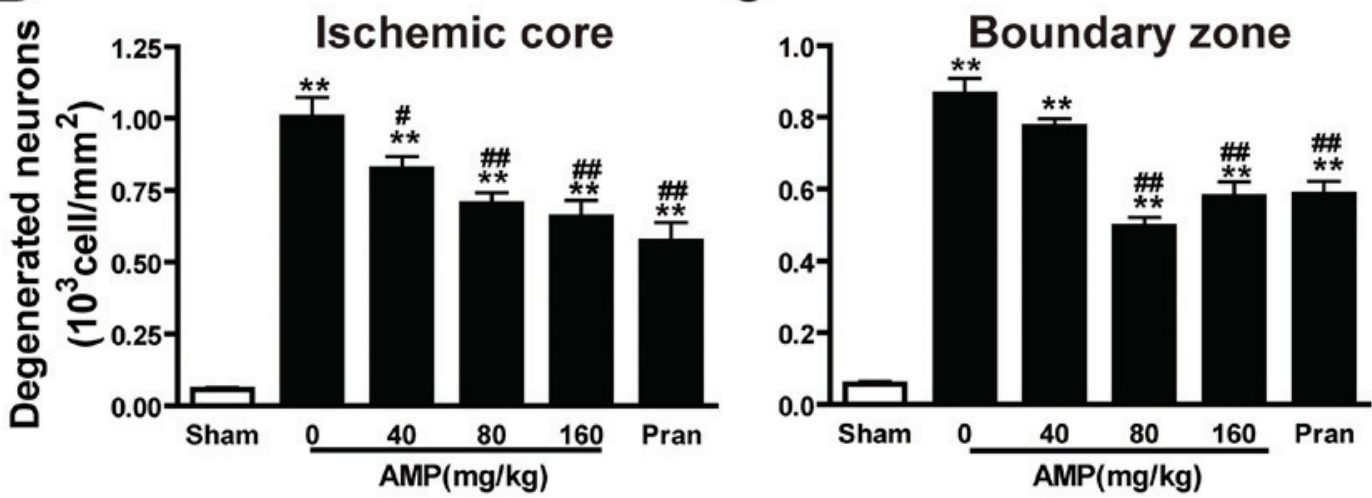

Figure 5. Effects of AMP and pran on the density of degenerated neurons in cortex $24 \mathrm{~h}$ following middle cerebral artery occlusion in rats. Brain sections were stained with Fluoro-Jade B fluorescent dye to detect degenerated neurons $24 \mathrm{~h}$ following reperfusion. (A) AMP (160 mg/kg, p.o.) and pran (0.1 mg/kg, i.p.) reduced the density of Fluoro-Jade B-stained neurons in the ischemic core (top panels) and boundary zone (bottom panels). Scale bar, $100 \mu \mathrm{m}$. Numbers of Fluoro-Jade B-stained neurons in (B) the ischemic core and (C) boundary zone are summarized. AMP (80 and $160 \mathrm{mg} / \mathrm{kg}, \mathrm{p} . \mathrm{o}$.$) and pran (0.1 mg / \mathrm{kg}$, i.p.) significantly reduced the number of degenerated neurons of the ischemic core and boundary zones $(\mathrm{n}=6) .{ }^{* * *} \mathrm{P}<0.01$ vs. sham; ${ }^{*} \mathrm{P}<0.05$ and ${ }^{\# \#} \mathrm{P}<0.01 \mathrm{vs}$. ischemic control, analyzed by one-way analysis of variance. AMP, ampelopsin; Pran, pranlukast.

by macrophages following the effect of D-galactosamine $(9,21)$. In PC12 cells, AMP inhibits $\mathrm{H}_{2} \mathrm{O}_{2}$-induced apoptosis via ERK and Akt signaling pathways, which are markedly involved in inflammatory cascades (9). The present study confirms that AMP inhibited the increase of MCAO-induced IL-1 $\beta$ and TNF- $\alpha$ in serum and CSF, while decreasing IgG exudation. TNF- $\alpha$ is a key inflammatory mediator in the altered permeability of BBB during reperfusion (39). TNF- $\alpha$ caused cramps in the microartery and increased the permeability and IL-1 $\beta$ caused an inflammatory reaction by promoting the adherence of leukocytes to endothelial cells, resulting in BBB injury $(40,41)$. Therefore, the current study concluded that AMP exhibited neuroprotective effects via its anti-inflammatory activity and increased resistance to $\mathrm{BBB}$ damage.

Ischemic stroke triggers a highly interconnected and complex cascade of cellular and molecular events in three phases: Acute (min to h), subacute (h to days) and chronic (days to months) $(42,43)$. In the acute phase, neuronal injury is predominantly due to ion imbalance, oxidative stress, energy failure, excitotoxicity and BBB dysfunction. In the late (subacute/chronic) phases, reactive astrocytosis and glial scar formation are critical responses, and are associated with beneficial effects including, restoration of astrocyte reactivity, de novo expression of glial proteins and process enlarging and detrimental effects, including disrupted planar cell polarity and slower ventricular movement (42-44). In the present study, AMP was demonstrated to attenuate neuronal injury by improving BBB breakdown in the acute phase. It was previously indicated that AMP attenuated oxygen-glucose deprivation/recovery injury in rat astrocytes (data not published), which suggests that AMP may protect rats against chronic ischemic brain injury. Future studies are required to elucidate the effects of AMP in the late phase of ischemic injury, along with the underlying mechanisms. 

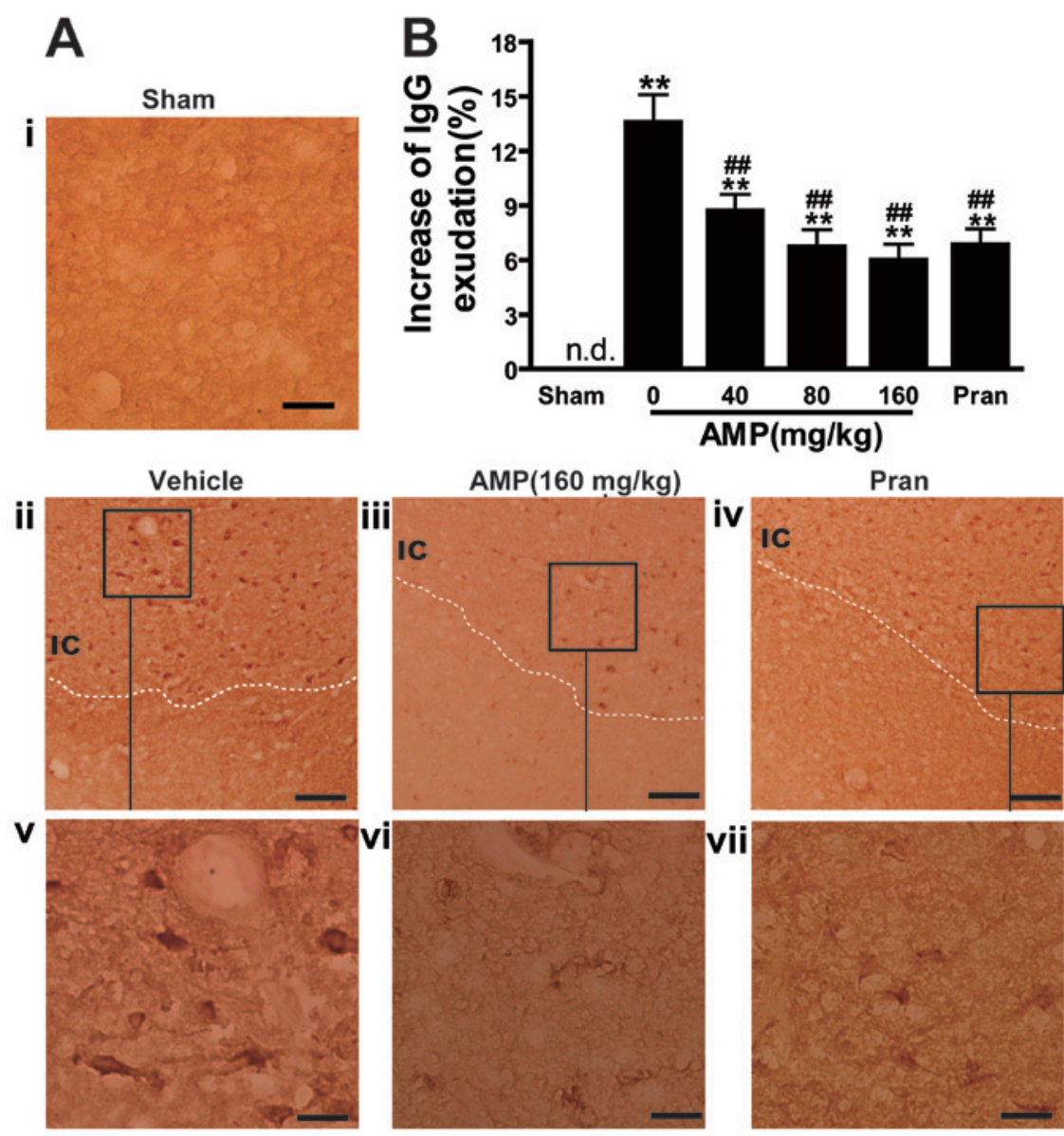

Figure 6. Effect of AMP and pran on IgG exudation in ischemic hemisphere $24 \mathrm{~h}$ following middle cerebral artery occlusion in rats. (A) Representative images of IgG-immunostained positive cells demonstrated that IgG exudation $24 \mathrm{~h}$ following reperfusion was attenuated by AMP (160 mg/kg, p.o.) and pran $(0.1 \mathrm{mg} / \mathrm{kg}$, i.p.). Scale bars, $100 \mu \mathrm{m}$ (i-iv) and, $25 \mu \mathrm{m}$ (v-vii). (B) Percentage increase of gray-scales of the injured hemisphere. AMP (80 and $160 \mathrm{mg} / \mathrm{kg}, \mathrm{p} .0$.) and pran $\left(0.1 \mathrm{mg} / \mathrm{kg}\right.$, i.p.) inhibited $\mathrm{IgG}$ exudation $(\mathrm{n}=6) .{ }^{* *} \mathrm{P}<0.01 \mathrm{vs}$. sham, ${ }^{\# \#} \mathrm{P}<0.01 \mathrm{vs}$. ischemic control, analyzed by one-way analysis of variance. AMP, ampelopsin; Pran, pranlukast; IgG, immunoglobulin G; n.d. not detectable.
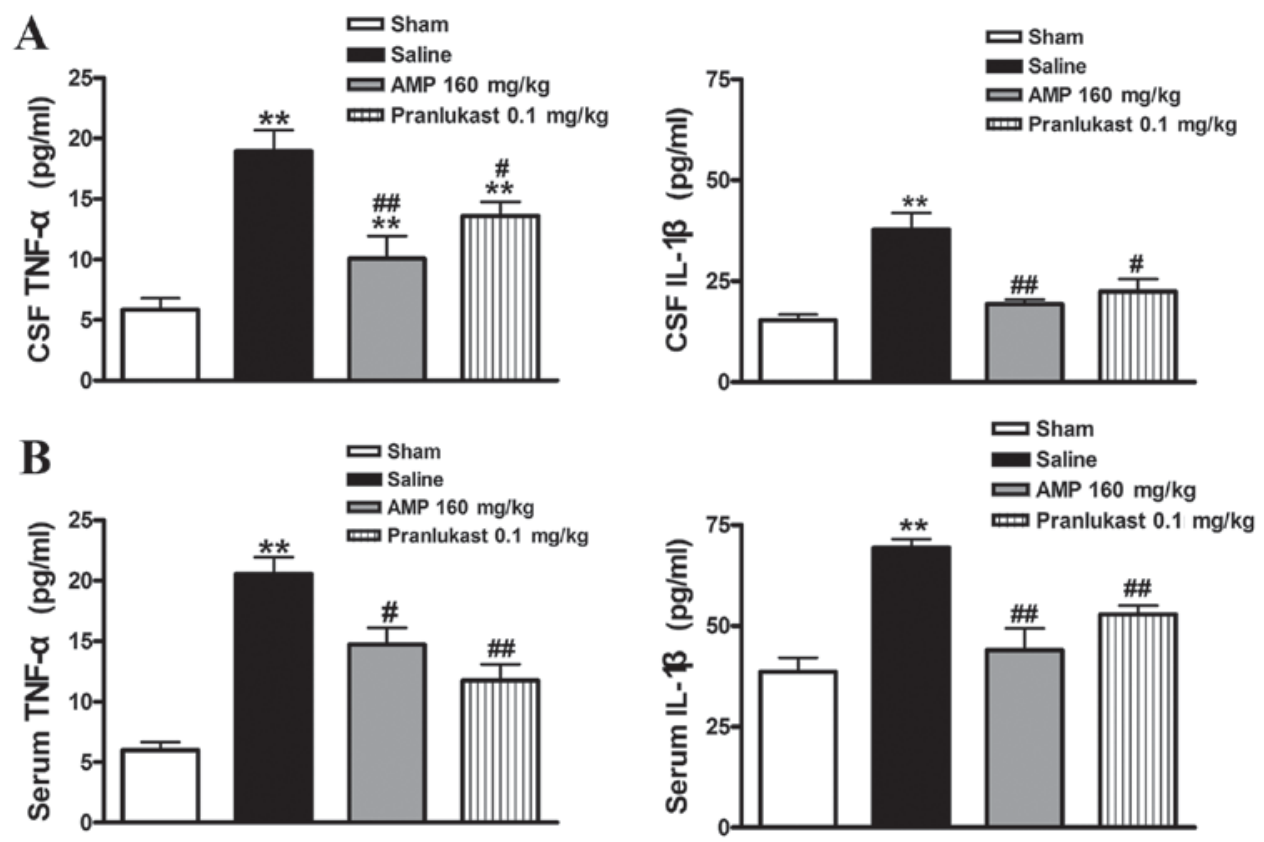

Figure 7. Effects of AMP and pranlukast on the level of cytokines in serum and CSF $24 \mathrm{~h}$ following MCAO in rats. Cytokine levels in (A) serum and (B) cerebrospinal fluid were assessed $24 \mathrm{~h}$ following MCAO. AMP $(80$ and $160 \mathrm{mg} / \mathrm{kg}$, p.o.) and pranlukast $(0.1 \mathrm{mg} / \mathrm{kg}$, i.p.) significantly inhibited the release of IL-1 $\beta$ and TNF- $\alpha$ in serum and CSF $(n=6) .{ }^{* *} \mathrm{P}<0.01$ vs. sham; ${ }^{*} \mathrm{P}<0.05$ and ${ }^{\# \#} \mathrm{P}<0.01$ vs. ischemic control (saline); analyzed by one-way analysis of variance. AMP, ampelopsin; MCAO, middle cerebral artery occlusion; CSF, cerebrospinal fluid; IL-1 $\beta$, interleukin- $1 \beta$; TNF- $\alpha$, tumor necrosis factor- $\alpha$. 
In conclusion, the present study demonstrated that oral administration of AMP mitigates acute brain injury following focal cerebral ischemia in rats, similar to the effect of the anti-inflammatory agent pranlukast. The results of the current study suggest that AMP may represent a novel class of therapeutic agent in the treatment of ischemic stroke. However, the neuroprotective effects of AMP were only demonstrated in rats, and therefore the detailed mechanisms underlying the effects of AMP in ischemic brain injury require further investigation.

\section{Acknowledgements}

The present study was supported by the National Natural Science Foundation of China (grant nos. 31301933 and 81401566), the Zhejiang Provincial Natural Science Foundation of China (grant no. Q15H090023), the Science and Technology Planning Project in Zhejiang Province (grant no. 2014C37011) and the Medical Science and Technology Planning Project Zhejiang Province (grant no. 201477310).

\section{References}

1. Tsai CF, Thomas B and Sudlow CL: Epidemiology of stroke and its subtypes in Chinese vs white populations: A systematic review. Neurology 81: 264-272, 2013.

2. Pereira VM, Yilmaz H, Pellaton A, Slater LA, Krings T and Lovblad KO: Current status of mechanical thrombectomy for acute stroke treatment. J Neuroradiol 42: 12-20, 2015.

3. Pierot L, Soize S, Benaissa A and Wakhloo AK: Techniques for endovascular treatment of acute ischemic stroke: From intra-arterial fibrinolytics to stent-retrievers. Stroke 46: 909-914, 2015.

4. Kou X and Chen N: Pharmacological potential of ampelopsin in Rattan tea Food Sci Human Wellness 1: 14-18, 2012

5. Murakami T, Miyakoshi M, Araho D, Mizutani K, Kambara T, Ikeda T, Chou WH, Inukai M, Takenaka A and Igarashi K: Hepatoprotective activity of tocha, the stems and leaves of Ampelopsis grossedentata, and ampelopsin. Biofactors 21: 175-178, 2004

6. Tahara S: A journey of twenty-five years through the ecological biochemistry of flavonoids. Biosci Biotechnol Biochem 71: 1387-1404, 2007.

7. Klotter F and Studer A: Total synthesis of resveratrol-based natural products using a palladium-catalyzed decarboxylative arylation and an oxidative Heck reaction. Angew Chem Int Ed Engl 53: 2473-2476, 2014.

8. Pflieger A, Waffo Teguo P, Papastamoulis Y, Chaignepain S, Subra F, Munir S, Delelis O, Lesbats P, Calmels C, Andreola ML, et al: Natural stilbenoids isolated from grapevine exhibiting inhibitory effects against HIV-1 integrase and eukaryote MOS1 transposase in vitro activities. PLoS One 8: e81184, 2013.

9. Qi S, Xin Y, Guo Y, Diao Y, Kou X, Luo L and Yin Z: Ampelopsin reduces endotoxic inflammation via repressing ROS-mediated activation of PI3K/Akt/NF-kB signaling pathways. Int Immunopharmacol 12: 278-287, 2012.

10. Kim JY, Jeong HY, Lee HK, Kim S, Hwang BY, Bae K and Seong YH: Neuroprotection of the leaf and stem of Vitis amurensis and their active compounds against ischemic brain damage in rats and excitotoxicity in cultured neurons. Phytomedicine 19: 150-159, 2012.

11. Zhou Y, Shu F, Liang X, Chang H, Shi L, Peng X, Zhu J and Mi M: Ampelopsin induces cell growth inhibition and apoptosis in breast cancer cells through ROS generation and endoplasmic reticulum stress pathway. PLoS One 9: e89021, 2014.

12. Kou X, Shen K, An Y, Qi S, Dai WX and Yin Z: Ampelopsin inhibits $\mathrm{H}_{2} \mathrm{O}_{2}$-induced apoptosis by ERK and Akt signaling pathways and up-regulation of heme oxygenase- 1 . Phytother Res 26 : 988-994, 2012

13. Zhang B, Dong S, Cen X, Wang X, Liu X, Zhang H, Zhao X and $\mathrm{Wu} \mathrm{Y}$ : Ampelopsin sodium exhibits antitumor effects against bladder carcinoma in orthotopic xenograft models. Anticancer Drugs 23: 590-596, 2012
14. Dung HV, Cuong TD, Chinh NM, Quyen D, Kim JA, Byeon JS, Woo MH, Choi JS and Min BS: Compounds from the aerial parts of Piper bavinum and their anti-cholinesterase activity. Arch Pharm Res 38: 677-682, 2015.

15. Papastamoulis Y, Richard T, Nassra M, Badoc A, Krisa S, Harakat D, Monti JP, Mérillon JM and Waffo-Teguo P: Viniphenol A, a complex resveratrol hexamer from Vitis vinifera stalks: Structural elucidation and protective effects against amyloid- $\beta$-induced toxicity in PC12 cells. J Nat Prod 77: 213-217, 2014.

16. Lambertsen KL, Biber K and Finsen B: Inflammatory cytokines in experimental and human stroke. J Cereb Blood Flow Metab 32: 1677-1698, 2012

17. Shi QJ, Wang H, Liu ZX, Fang SH, Song XM, Lu YB, Zhang WP, Sa XY, Ying HZ and Wei EQ: HAMI 3379, a CysLT2R antagonist, dose- and time-dependently attenuates brain injury and inhibits microglial inflammation after focal cerebral ischemia in rats. Neuroscience 291: 53-69, 2015.

18. Xing C, Arai K, Lo EH and Hommel M: Pathophysiologic cascades in ischemic stroke. Int J Stroke 7: 378-385, 2012.

19. Turner RC, Dodson SC, Rosen CL and Huber JD: The science of cerebral ischemia and the quest for neuroprotection: Navigating past failure to future success. J Neurosurg 118: 1072-1085, 2013

20. Kim HH, Oh MH, Park KJ, Heo JH and Lee MW: Anti-inflammatory activity of sulfate-containing phenolic compounds isolated from the leaves of Myrica rubra. Fitoterapia 92: 188-193, 2014

21. Ku KT, Huang YL, Huang YJ and Chiou WF: Miyabenol A inhibits LPS-induced NO production via IKK/IkappaB inactivation in RAW 264.7 macrophages: Possible involvement of the p38 and PI3K pathways. J Agric Food Chem 56: 8911-8918, 2008.

22. Shi QJ, Xiao L, Zhao B, Zhang XY, Wang XR, Xu DM, Yu SY, Fang SH, Lu YB, Zhang WP, et al: Intracerebroventricular injection of HAMI 3379, a selective cysteinyl leukotriene receptor 2 antagonist, protects against acute brain injury after focal cerebral ischemia in rats. Brain Res 1484: 57-67, 2012.

23. Chu LS, Wei EQ, Yu GL, Fang SH, Zhou Y, Wang ML and Zhang WP: Pranlukast reduces neutrophil but not macrophage/microglial accumulation in brain after focal cerebral ischemia in mice. Acta Pharmacol Sin 27: 282-288, 2006.

24. Yu GL, Wei EQ, Wang ML, Zhang WP, Zhang SH, Weng JQ, Chu LS, Fang SH, Zhou Y, Chen Z, et al: Pranlukast, a cysteinyl leukotriene receptor-1 antagonist, protects against chronic ischemic brain injury and inhibits the glial scar formation in mice. Brain Res 1053: 116-125, 2005.

25. Zhang LH and Wei EQ: Neuroprotective effect of ONO-1078, a leukotriene receptor antagonist, on transient global cerebral ischemia in rats. Acta Pharmacol Sin 24: 1241-1247, 2003.

26. Longa EZ, Weinstein PR, Carlson S and Cummins R: Reversible middle cerebral artery occlusion without craniectomy in rats. Stroke 20: 84-91, 1989.

27. Nirogi R, Kandikere V,Mudigonda K, Bhyrapuneni G, Muddana N, Saralaya R and Benade V: A simple and rapid method to collect the cerebrospinal fluid of rats and its application for the assessment of drug penetration into the central nervous system. J Neurosci Methods 178: 116-119, 2009

28. Pegg CC, He C, Stroink AR, Kattner KA and Wang CX: Technique for collection of cerebrospinal fluid from the cisterna magna in rat. J Neurosci Methods 187: 8-12, 2010.

29. Yonemori F, Yamaguchi T, Yamada $\mathrm{H}$ and Tamura A: Evaluation of a motor deficit after chronic focal cerebral ischemia in rats. J Cereb Blood Flow Metab 18: 1099-1106, 1998.

30. Lin TN, He YY, Wu G, Khan M and Hsu CY: Effect of brain edema on infarct volume in a focal cerebral ischemia model in rats. Stroke 24: 117-121, 1993.

31. Schmued LC and Hopkins KJ: Fluoro-Jade B: A high affinity fluorescent marker for the localization of neuronal degeneration. Brain Res 874: 123-130, 2000.

32. Schmidt-Kastner R, Meller D, Bellander BM, Strömberg I, Olson L and Ingvar M: A one-step immunohistochemical method for detection of blood-brain barrier disturbances for immunoglobulins in lesioned rat brain with special reference to false-positive labelling in immunohistochemistry. J Neurosci Methods 46: 121-132, 1993.

33. Tominaga $\mathrm{T}$ and Ohnishi ST: Interrelationship of brain edema, motor deficits, and memory impairment in rats exposed to focal ischemia. Stroke 20: 513-518, 1989. 
34. Fang SH, Wei EQ, Zhou Y, Wang ML, Zhang WP, Yu GL, Chu LS and Chen Z: Increased expression of cysteinyl leukotriene receptor-1 in the brain mediates neuronal damage and astrogliosis after focal cerebral ischemia in rats. Neuroscience 140 969-979, 2006.

35. Gürer G, Gursoy-Ozdemir Y, Erdemli E, Can A and Dalkara T: Astrocytes are more resistant to focal cerebral ischemia than neurons and die by a delayed necrosis. Brain Pathol 19: 630-641, 2009.

36. Jia F, Yin YH, Gao GY, Wang Y, Cen L and Jiang JY: MMP-9 inhibitor SB-3CT attenuates behavioral impairments and hippocampal loss after traumatic brain injury in rat. J Neurotrauma 31: 1225-1234, 2014

37. Jiang Z, Watts LT, Huang S, Shen Q, Rodriguez P, Chen C, Zhou C and Duong TQ: The effects of methylene blue on autophagy and apoptosis in MRI-defined normal tissue, ischemic penumbra and ischemic core. PLoS One 10: e0131929, 2015

38. Gotoh O, Asano T, Koide $\mathrm{T}$ and Takakura K: Ischemic brain edema following occlusion of the middle cerebral artery in the rat. I: The time courses of the brain water, sodium and potassium contents and blood-brain barrier permeability to $125 \mathrm{I}$-albumin Stroke 16: 101-109, 1985.
39. Lv S, Song HL, Zhou Y, Li LX, Cui W, Wang W and Liu P: Tumour necrosis factor-alpha affects blood-brain barrier permeability and tight junction-associated occludin in acute liver failure. Liver Int 30: 1198-1210, 2010.

40. Rodrigues SF and Granger DN: Blood cells and endothelial barrier function. Tissue Barriers 3: e978720, 2015.

41. Willis CL, Brooks TA and Davis TP: Chronic inflammatory pain and the neurovascular unit: A central role for glia in maintaining BBB integrity? Curr Pharm Des 14: 1625-1643, 2008.

42. Candelario-Jalil E: Injury and repair mechanisms in ischemic stroke: Considerations for the development of novel neurotherapeutics. Curr Opin Investig Drugs 10: 644-654, 2009.

43. Kriz J: Inflammation in ischemic brain injury: Timing is important. Crit Rev Neurobiol 18: 145-157, 2006.

44. Jørgensen HS, Nakayama H, Raaschou HO and Olsen TS: Progressive apoplexy. Incidence, risk factors and prognosis-the Copenhagen stroke study. Ugeskr Laeger 157: 3619-3622, 1995 (In Danish). 